\title{
The double cantilever beam test applied to mode I fracture characterization of cortical bone tissue
}

\author{
J.J.L. Morais ${ }^{a}$, M.F.S.F. de Moura ${ }^{b, *}$, F.A.M. Pereira ${ }^{a}$, J. Xavier ${ }^{a}$, N. Dourado $^{a}$, M.I.R. Dias ${ }^{c}$, \\ J.M.T. Azevedo ${ }^{d}$ \\ a CITAB/UTAD, Departamento de Engenharias, Quinta de Prados, 5001-801, Vila Real, Portugal

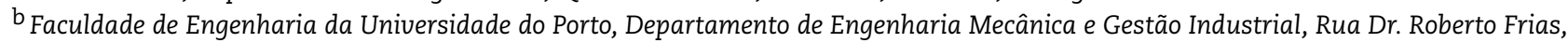 \\ 4200-465 Porto, Portugal \\ C UTAD, Departamento de Ciências Veterinárias, Quinta de Prados, 5001-801, Vila Real, Portugal \\ d CECAV/UTAD, Departamento de Zootecnia, Quinta de Prados, 5001-801, Vila Real, Portugal
}

\section{A R T I C L E I N F O}

\section{Article history:}

Received 9 January 2010

Received in revised form

26 March 2010

Accepted 1 April 2010

Published online 14 April 2010

Keywords:

Bone

Compliance

Fracture toughness

Modeling

\begin{abstract}
A B S T R A C T
The primary objective of this work was to analyse the adequacy of the Double Cantilever Beam (DCB) test in determining fracture toughness under pure mode I loading of cortical bone tissue. A new data reduction scheme based on specimen compliance and the crack equivalent concept was used to overcome the difficulties inherent in crack monitoring during its growth. It provides a complete resistance curve, which is fundamental in estimating the fracture energy. A cohesive zone model was used to simulate damage initiation and propagation, thus assessing the efficacy of the proposed testing method and data reduction scheme. Subsequently, the DCB test was applied to evaluate the mode I fracture energy of hydrated and thermally dehydrated cortical bone tissue from young bovine femur, in the tangential-longitudinal propagation system. The results obtained demonstrate the efficacy of the DCB test and the proposed data reduction scheme on the bone fracture characterization under mode I loading.
\end{abstract}

(C) 2010 Elsevier Ltd. All rights reserved.

\section{Introduction}

Cortical bone is a composite material comprising mineral (mainly hydroxyapatite) and organic (mostly type I collagen) phases, arranged in a complex hierarchical, heterogeneous and anisotropic microstructure (Rho et al., 1998). The quality of organic and mineral phases, as well as their spatial arrangement and interaction, contribute to the mechanical properties of the material. It is accepted that the mineral phase is the main contributor to the elastic and strength properties of cortical bone (Currey, 1988b; Hernandez et al., 2001), whereas the organic phase predominantly determines the viscoelastic properties (Bowman et al., 1999) and toughness (Zioupos et al., 1999; Wang et al., 2000, 2002). The other main phase of cortical bone is water, and early studies demonstrate its crucial role in determining the mechanical behavior of bone tissue. The dehydration of cortical bone tissue increases the modulus of elasticity and the tensile strength, but decreases the toughness (Currey, 1988a; Lucksanasombool et al., 2001; Nyman et al., 2006; Wang

\footnotetext{
* Corresponding author.

E-mail address: mfmoura@fe.up.pt (M.F.S.F. de Moura).
} 
and Agrawal, 1996). Also, dehydration affects the viscoelastic behaviour of cortical bone: for instance, it reduces the loss factor without appreciable changes in the storage modulus (Yamashita et al., 2002), and increases the relaxation rate (Sasaki and Enyo, 1995).

Because in vivo bone fractures are often initiated and/or promoted by cracks, Fracture Mechanics emerges as an essential tool to assess bone tissue quality, in order to improve the diagnoses of fracture risks and the treatment of bone diseases (Wang and Puram, 2004). To date, most assessments of the toughness of cortical bone have involved linear-elastic fracture mechanics (LEFM), which yield a singlevalued fracture parameter: the critical stress intensity factor or the critical energy release rate (Norman et al., 1995, 1996). The majority of the works have focused on the resistance to pre-crack starting advance under tension, both in the longitudinal (Norman et al., 1995, 1996) and transverse (Phelps et al., 2000) axes of long bones. A variety of fracture test specimens has been used: the compact tension (CT) specimen (Norman et al., 1995); the chevron-notched beam specimen (Yan et al., 2006); the compact sandwich tension specimen (Wang and Agrawal, 1996); and the single-edge notched beam (SEN) specimen (Phelps et al., 2000). A wide range of critical stress intensity factors or critical energy release rates for cortical bone has been reported, for the very reason that they vary depending on the source, type and location of bone tested, as well as the testing method (Yan et al., 2006; Brown et al., 2000).

The LEFM approach provides a limited insight into fracture behaviour of cortical bone. Indeed, as a consequence of its composition and microstructure, several toughening mechanisms have been observed in the fracture of cortical bone, such as diffuse microcracking, crack deflection, and fibre bridging (Zioupos, 1998; Vashishth et al., 2000; Yeni and Norman, 2000; Nalla et al., 2003). These toughening mechanisms are responsible for the resistance curve (Rcurve) behaviour observed by several researchers (Malik et al., 2003; Vashishth, 2004; Nalla et al., 2004b). This fact, allied to the size-scales involved in fracture testing of cortical bone, renders the pure LEFM theory inexact when applied to this material (Lucksanasombool et al., 2001; Ural and Vashishth, 2006). In the light of this, several authors have recently applied cohesive zone models for analyzing initiation and propagation of cracks in cortical bone (Ural and Vashishth, 2006; Yang et al., 2006; Cox and Yang, 2007). However, a number of issues associated with this approach are yet unresolved, including the test methods and the data reduction methodologies more appropriate for accurate identification of the cohesive law. The cohesive zone models, integrated in finite element analysis, have a great potential inasmuch as they allow analysing the influence of several factors controlling fracture initiation and propagation of cracks in cortical bone.

The aim of this work is to analyse the adequacy of the Double Cantilever Beam (DCB) test in assessing the fracture energy under pure mode I loading of cortical bone tissue. This test presents several advantages relative to other geometries (e.g., CT, SEN). In fact, the beam theory can be applied thus providing user-friendly data reduction methods for toughness measurements. In addition, more stable crack growth takes place and a longer region of damage zone development exists, thus providing adequate self-similar crack propagation and consequently avoiding spurious effects on the fracture energy measurements.

A new data reduction scheme is proposed owing to the difficulty associated to monitoring the crack length in this material. This method is based on the specimen compliance and the crack equivalent concept. It does not require crack length measurements during its propagation and provides a complete $\mathrm{R}$-curve, which is fundamental to estimating the fracture energy issued from its plateau. A cohesive zone model was used to simulate damage initiation and propagation thus assessing the efficacy of the proposed testing method and data reduction scheme. Subsequently, the DCB test was employed to evaluate the mode I fracture energy of hydrated and thermally dehydrated cortical bone tissue from bovine femora of young animals, in the tangential-longitudinal propagation system (i.e., the normal to the crack plane is the tangential direction of mid-diaphysis and the crack propagation direction is the longitudinal direction of mid-diaphysis). The achievements of this work demonstrate that the DCB test can be applied with advantages to fracture characterization of bone under mode I loading.

\section{Data reduction scheme}

The classical data reduction schemes used to determine the fracture energy in mode I $\left(G_{\text {IC }}\right.$ ) require crack-length monitoring during the fracture tests, which is difficult to perform with sufficient accuracy in bone. The compliance calibration method is based on the Irwin-Kies relation

$G_{I}=\frac{P^{2}}{2 B} \frac{d C}{d a}$

which depends on the compliance $(C=\delta / P)$ calibration as a function of the crack length a (Fig. 1). On the other hand, the corrected beam theory is expressed through the following equation (ISO 15024, 2001):

$\mathrm{G}_{I}=\frac{3 \mathrm{P} \delta}{2 \mathrm{~B}(a+|\Delta|)}$

where $\Delta$ is an experimentally determined correction term. In this method the crack-length is also a fundamental parameter. However, the monitoring of $a$ is a complicated task (Fig. 2). Moreover, it is known that a non-negligible fracture process zone (FPZ) can develop ahead of the crack tip, as a consequence of the nucleation of multiple micro-cracks (the intrinsic toughening mechanism according to Nalla et al. (2004a)), and in the crack wake, as a consequence of crack bridging due to uncracked ligaments and collagen fibres (the extrinsic crack mechanism according to Nalla et al. (2004a)). These non-linear processes lead to an energy dissipation, which is not accounted for when the real crack-length is used. As a consequence, an alternative data reduction scheme based on an equivalent crack as a function of current specimen compliance is proposed. 


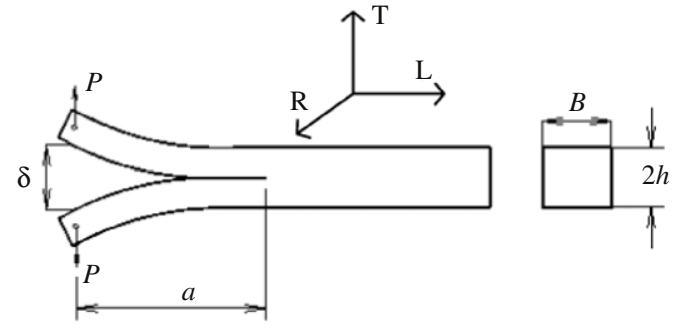

Fig. 1 - Sketch of the Double Cantilever Beam (DCB) test (L is the longitudinal direction, $R$ is the radial direction, and $T$ is the tangential direction of the femur).

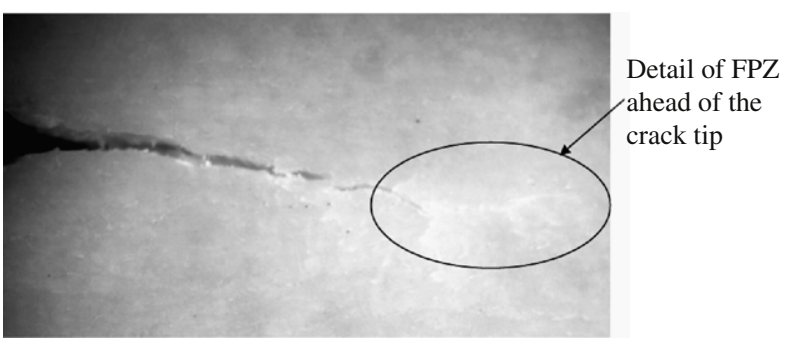

Fig. 2 - Crack-tip detail in bone.

\section{Compliance based beam method}

The Compliance Based Beam Method (CBBM) uses the Timoshenko beam theory to establish the $C=f(a)$ relationship (de Moura et al., 2008)

$C=\frac{8 a^{3}}{E_{L} B h^{3}}+\frac{12 a}{5 B h G_{L T}}$

where $E_{L}$ is the longitudinal elastic modulus and $G_{L T}$ the shear modulus in the TL plane (Fig. 1). This equation can be used to estimate the equivalent crack length as a function of the current compliance $C$ computed directly from the measured load-displacement $(P-\delta)$ curve, i.e. $a_{e}=$ $f(C)$. The solution of this equation is obtained using the Matlab ${ }^{\circledR}$ software (see Appendix). Following this procedure the effect of the FPZ is taken into account, since its presence affects the current compliance. The combination of Eqs. (1) and (3) leads to

$G_{I}=\frac{6 P^{2}}{B^{2} h}\left(\frac{2 a_{e}^{2}}{E_{L} h^{2}}+\frac{1}{5 G_{L T}}\right)$.

Using this method a complete R-curve is determined without the direct measurement of $a$. Another relevant aspect is related to the non-negligible scatter in the elastic modulus observed in bone (Rho et al., 1998). To overcome this issue an inverse method could be employed. The initial (elastic) compliance measured from the experimental $\mathrm{P}-\delta$ curves can be used to estimate the longitudinal modulus of each specimen through a fitting procedure. Therefore, each specimen should be numerically simulated (Finite Element analysis) taking into account its real dimensions. Several iterations (normally two or three) may be necessary till the numerical initial compliance agrees with the experimental one, thus defining the specimen modulus $\left(E_{L}\right)$.

\section{Numerical validation}

The validation of the proposed data reduction scheme, i.e. the CBBM described in the previous section, was performed through numerical analysis considering the Finite Element (FE) method including a cohesive zone model (CZM). The material properties used in the numerical simulations were obtained from Dempster and Coleman (1961) and Dong and Guo (2004). The fracture energy inputted ( $G_{\text {Ic }}$ inp) in the CZM is a key parameter in the model validation. In the numerical simulations a value of $2.0 \mathrm{~N} / \mathrm{mm}$, which is close to that obtained in the present experiments for hydrated bone, was used. The load-displacement curve obtained during damage initiation and growth is used to estimate the R-curve. The validation procedure consists of verifying if the data reduction scheme presented above is able to reproduce, in the plateau of the $R$-curve, the nominal value of the fracture energy inputted in the model ( $G_{\text {IC }}$ inp).

\subsection{Cohesive zone model}

The 6-node two-dimensional interface finite element was used to simulate damage onset and propagation in the DCB test using the ABAQUS ${ }^{\circledR}$ software, connected with the solid in-plane stress 8-node element. The cohesive zone model (CZM) is based on a stress-relative displacement $\left(\sigma_{i}-w_{i}\right.$, with $i=$ I, II) softening law represented in Fig. 3 (Ural and Vashishth, 2006; Morais et al., 2003). This law requires the definition of two cohesive parameters (possible to be determined experimentally), i.e. the local strength $\sigma_{u, i}$ and the respective fracture energy $G_{i c}$ which corresponds to the area of the major triangle in Fig. 3. The relative displacement $w_{o, i}$ is calculated as a function of the local ultimate stress $\sigma_{u, i}$ and the stiffness value (normal or shear) at the interface. The optimum initial stiffness value is defined as being the highest value which does not induce numerical problems. Most of the structural problems have been solved for $10^{6}$ to $10^{8} \mathrm{~N} / \mathrm{m}^{3}$ (de Moura et al., 1997). The mixed mode law (I/II) is an extension of the pure mode I and assumes a quadratic stress criterion including the two modes to simulate damage initiation

$\left(\frac{\sigma_{I}}{\sigma_{u, I}}\right)^{2}+\left(\frac{\sigma_{I I}}{\sigma_{u, I I}}\right)^{2}=1 \quad$ if $\sigma_{I} \geq 0$

$\sigma_{\text {II }}=\sigma_{\text {U,II }}$ if $\sigma_{\mathrm{I}} \leq 0$

where it is assumed that normal compressive stresses do not contribute to damage onset. On the other hand, crack propagation is simulated through the linear energetic criterion,

$\frac{G_{I}}{G_{I c}}+\frac{G_{I I}}{G_{I I c}}=1$.

It should be emphasized that this mixed mode law has wide general application, since the pure mode loading cases (I or II) are particular cases of the mixed mode formulation. The model is detailed in de Moura et al. (2008).

\subsection{Numerical results}

In order to verify the adequacy of the data reduction scheme, the DCB specimen was simulated taking into account the 


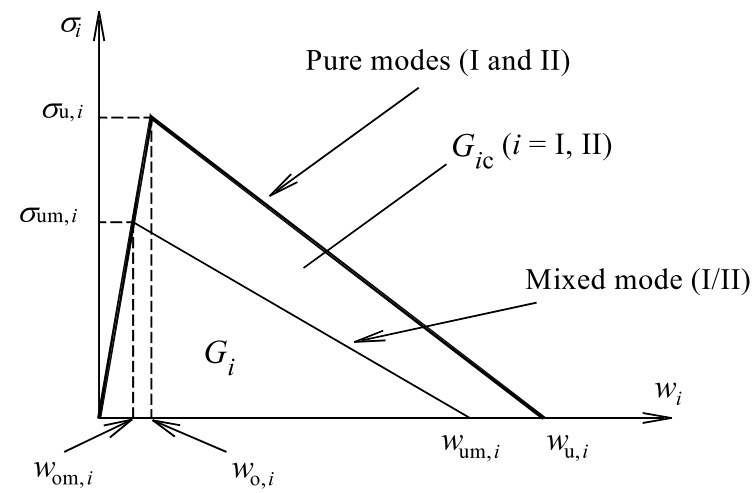

Fig. 3 - Sketch of the pure (I or II) and mixed (I/II) bilinear cohesive zone model.

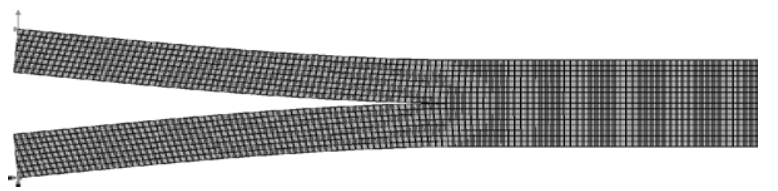

Fig. 4 - Mesh used in the DCB simulation.

Table 1 - Nominal mechanical properties of cortical bone (Dempster and Coleman, 1961; Dong and Guo, 2004).

\begin{tabular}{lllll}
$E_{L}(\mathrm{GPa})$ & $E_{\mathrm{T}}(\mathrm{GPa})$ & $\mathrm{G}_{\mathrm{LT}}(\mathrm{GPa})$ & $\nu_{\mathrm{LT}}$ & $\sigma_{u, I}(\mathrm{GPa})$ \\
\hline 16.61 & 9.55 & 4.74 & 0.37 & 20.0 \\
\hline
\end{tabular}

cohesive mixed mode zone model described above. Fig. 4 shows the refined FE mesh used with 7680 two-dimensional plane solid elements and 480 interface finite elements with null thickness located along the crack path (half-height of the specimen). A non-linear geometrical analysis was considered and a very small increment size was used (0.005 of the applied displacement) to guarantee smooth crack growth. Nominal properties presented in Table 1 were used (Dempster and Coleman, 1961; Dong and Guo, 2004). Fig. 5(a) presents the plot of the load-displacement $(P-\delta)$ curve. The corresponding $R$-curve obtained by means of the CBBM described in Section 2 shows excellent agreement on its plateau with the inputted fracture energy (see Fig. 5(b)). This important result demonstrates that the proposed data reduction scheme is able to accurately provide the true fracture energy of the material being tested.

\section{Experiments}

Nineteen fresh bovine femora of young animals were acquired from a local slaughterhouse, within a one day postmortem period. The sex of the animals was not known and the ages were about 8 months. A longitudinal-transverse section was dissected from the medial aspect of the middiaphysis of each femur (Fig. 6) and immediately cleaned. From these sections a total of nineteen DCB specimens (one specimen from each section) with a length of $60 \mathrm{~mm}$
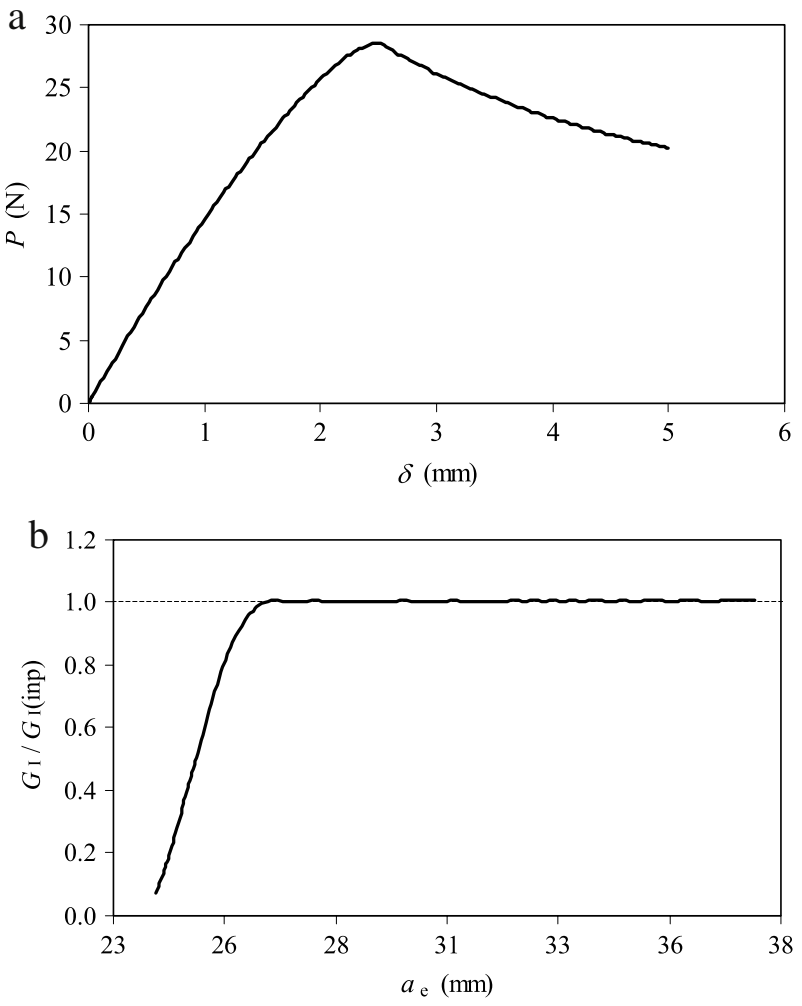

Fig. 5 - Numerical $P-\delta$ curve (a) and the corresponding R-curve (b).

were machined using a milling machine to get the nominal dimensions (Fig. 1): $B=2, h=3.5$ and $a_{0}=20$ (dimensions in $\mathrm{mm}$ ). During this process, the endosteal and periosteal tissues were removed. The initial crack length $a_{0}$ was introduced in two steps. First, a notch (1 mm thick) was machined using a circular saw. Then, a pre-crack (depth: $0.5-1.0 \mathrm{~mm}$ ) was created just before the fracture tests, by tapping a sharp razor blade into the notch. The specimens were orientated with the initial crack in the TL propagation system (where $T$ is the normal to the crack plane and $L$ is the crack propagation direction, Fig. 1) and the nominal crackgrowth direction along the proximal-distal direction of the femur. The specimens were kept moist during all steps of the machining process with physiological saline. Moreover, they were wrapped in gauze soaked in physiological saline, and frozen at $-20{ }^{\circ} \mathrm{C}$ for storage. The influence of water on the fracture properties of bone was assessed by testing hydrated and dehydrated bone samples. The hydrated sample (nine DCB specimens) was prepared by immersing the specimens in physiological saline at $37^{\circ} \mathrm{C}$, till constant weight (about $24 \mathrm{~h}$ ). The dehydrated sample (ten DCB specimens) was prepared from previously hydrated specimens, subsequently placed in a vacuum oven containing desiccant, at $37^{\circ} \mathrm{C}$ for approximately $48 \mathrm{~h}$. The weight fractions of the mineral and organic phases, as well as the water content, were determined by gravimetric analysis with an electronic balance (resolution of $0.1 \mathrm{mg}$ ). Small coupons (nominal dimensions: $2 \mathrm{~mm} \times$ $7 \mathrm{~mm} \times 7 \mathrm{~mm}$ ), matched with each DCB specimen before dehydration, were prepared. The hydrated coupons were weighed in air $\left(M_{w}\right)$ and submersed in distilled water $\left(M_{S}\right)$, 


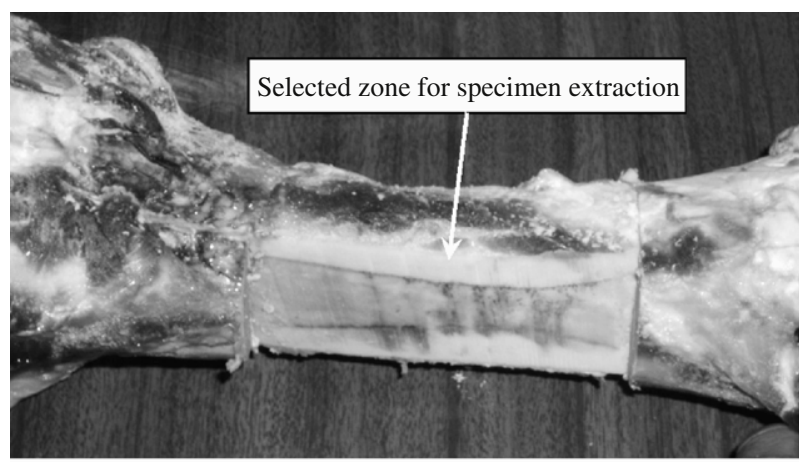

Fig. 6 - Mid-diaphysis of young bovine femur.

Table 2 - Cortical bone composition (19 specimens).

\begin{tabular}{lccll} 
& $\% \mathrm{M}_{\text {mineral }}$ & $\% \mathrm{M}_{\text {organic }}$ & $\% \mathrm{M}_{\text {water }}$ & $\% \mathrm{~V}_{\text {water }}$ \\
\hline Average & 62.00 & 28.06 & 9.94 & 18.42 \\
Cov $(\%)$ & 1.65 & 3.61 & 7.69 & 12.68 \\
\hline
\end{tabular}

and then dehydrated. The dry mass $\left(M_{d}\right)$ was measured at this stage. Afterwards, a furnace was used to ash the coupons at $800{ }^{\circ} \mathrm{C}$ for $4 \mathrm{~h}$. The mass of ash residue $\left(\mathrm{M}_{a}\right)$ was assumed to be the mass of mineral phase, and the difference between $M_{d}$ and $M_{a}$ was considered the mass of the organic phase. The mass fractions of the mineral, organic, and aqueous phases were then calculated in a wet basis, by dividing their masses by the mass of the hydrated coupon:

$\% M_{\text {water }}=\frac{M_{w}-M_{d}}{M_{w}} \times 100$

$\% M_{\text {mineral }}=\frac{M_{a}}{M_{w}} \times 100$

$\% M_{\text {organic }}=\frac{M_{d}-M_{a}}{M_{w}} \times 100$.

In addition, the volume fraction of water was determined as follows, according to the Archimedes principle:

$\% V_{\text {water }}=\frac{M_{w}-M_{d}}{M_{w}-M_{S}} \times 100$.

No significative differences were registered between the composition of wet and dry DCB samples. Thus, both data were pooled and presented in Table 2 .

A servo-electrical material testing system (MicroTester INSTRON 5848) was used to undergo fracture tests under displacement control and normal environmental conditions $\left(65 \% \mathrm{RH}\right.$ at $\left.20^{\circ} \mathrm{C}\right)$. A $2 \mathrm{kN}$ load-cell was installed and the displacement rate of the actuator was set to $0.5 \mathrm{~mm} / \mathrm{min}$. The acquisition frequency was set to $5 \mathrm{~Hz}$. The loading was applied to the specimen through a couple of piano hinges (Fig. 7), thus inducing a pure mode I loading. Piano hinges were bonded to the specimen using a fast curing epoxy adhesive. Fig. 2 shows the difficulty associated to the direct crack-length (a) measurement during damage progression.

The porosity of the specimens was measured by means of image processing and analysis. For that purpose, a slice of material was cut from each specimen after testing. These samples were embedded in a weakly exothermic epoxy and were ground using progressively finer grades of $\mathrm{SiC}$ paper, and

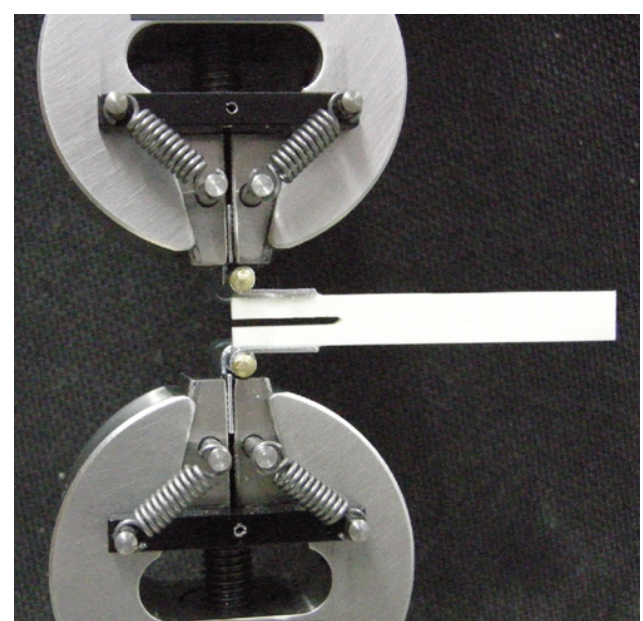

Fig. 7 - Photography showing the specimen detail.
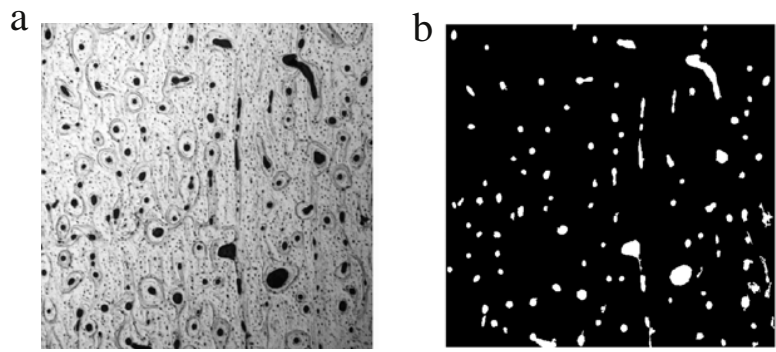

Fig. 8 - (a) Micrograph and (b) binary image of bone sample.

Table 3 - Cortical bone vascular porosity.

\begin{tabular}{lll} 
& Hydrated (\%) & Dehydrated (\%) \\
\hline Number of specimens & 9 & 10 \\
Average & 3.63 & 3.75 \\
Cov (\%) & 22.1 & 33.4 \\
\hline
\end{tabular}

lastly polished with $1 \mu \mathrm{m}$ diamond suspension embedded in soft polishing cloth. Both hydrated and dehydrated samples were analysed. Images of the samples were taken using an optical microscope coupled with an 8 bit CCD Dolphin camera (Allied Vision Technologies). The images were filtered and binary and morphology operations were applied to obtain a proper segmented image as shown in Fig. 8(a). A criterion of minimum area was chosen in order to ignore the canaliculi and lacuna for porosity measurements. The vascular porosity (i.e., the volume fraction of Harvesian canals and resorption cavities) was then estimated from the percentage of white area on the processed binary images (Fig. 8(b)). The results are reported in Table 3 . As can be seen, the vascular porosity of hydrated and dehydrated DCB samples are similar.

\section{Results and discussion}

The DCB test was applied to estimate the fracture energy of hydrated and dehydrated young bovine bone. Fig. 9 presents 

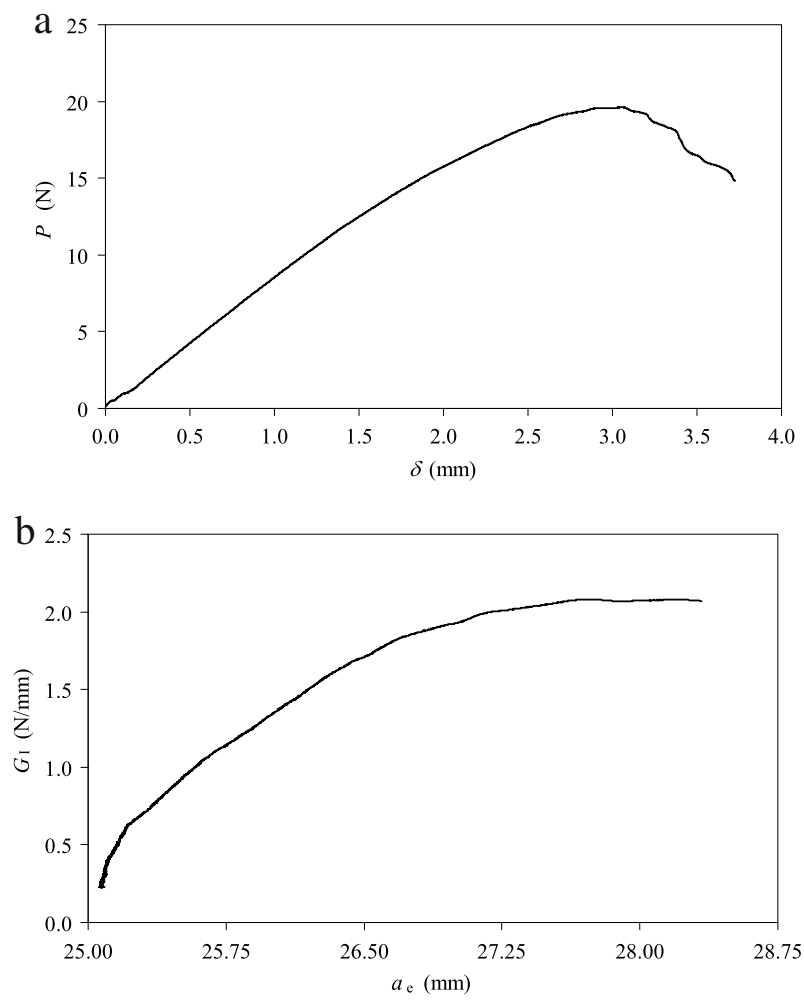

Fig. 9 - Typical P- $\delta(a)$ and the corresponding R-curve (b) for the hydrated bone.

the typical $\mathrm{P}-\delta$ and the corresponding $\mathrm{R}$-curve of the hydrated bone, obtained using the CBBM. The $\mathrm{P}-\delta$ curve (Fig. 9(a)) shows a pronounced non-linear behaviour near the peak load. This trend is explained by the existence of a pronounced fracture process zone (FPZ), as it can be seen in Fig. 2. The consequence of this softening process is a ductile behaviour which is reflected on the referred non-linear profile of the $\mathrm{P}-\delta$ curve. Consequently, the respective R-curve (Fig. 9(b)) shows a large ascending branch (approximately $3 \mathrm{~mm}$ of $a_{e}$ ) till the plateau value defining the material fracture energy $\left(G_{I c}\right)$ is attained. This extensive branch corresponds to the FPZ development up to its critical size, from which the selfsimilar crack growth takes place leading to conditions for an appropriate measurement of the material fracture energy. Fig. 10 shows the typical $P$ - $\delta$ curve and the related R-curve of the dehydrated bone. The $\mathrm{P}-\delta$ curve (Fig. 10(a)) shows a brittle behaviour up to the peak load. The associated R-curve (Fig. 10(b)) shows an almost vertical initial branch, which is explained by a small FPZ extent, thus also demonstrating that dehydrated bone became more brittle. In this more brittle material, it is observed (Fig. 10(b)) that the fracture energy at the crack initiation (beginning of the plateau) slightly increases relative to the corresponding value during its selfsimilar propagation (horizontal branch of the plateau). This phenomenon is induced by the crack blunting shape, which does not truly reproduce the real conditions of fracture. Indeed, since the radius of the crack tip is finite, the energy required to induce fracture initiation is artificially increased. It should be noted that this phenomenon is not visible in hydrated bone (Fig. 9(b)), as the large FPZ that develops at the crack-tip mitigates the referred spurious effect. Summing up, it should be highlighted that the proposed CBBM overcomes this drawback since it provides a complete $R$-curve, which allows the measurement of a consistent value of $G_{\text {Ic }}$ from the horizontal part of the plateau. Table 4 presents a summary of the elastic modulus and fracture energy obtained for nine hydrated specimens. It should be noted that the average toughness obtained $\left(G_{\text {Ic }}=1.91 \mathrm{~N} / \mathrm{mm}\right.$ ) is in the middle of the range $\left(1.388 \leq G_{\text {Ic }} \leq 2.557 \mathrm{~N} / \mathrm{mm}\right)$ presented in Doblaré et al. (2004). Table 5 exhibits similar results for ten dehydrated specimens. The coefficients of variation of both samples are acceptable for this type of material. Effectively, bone is a natural material exhibiting a high variability in its properties. As can be seen, dehydration causes an appreciable reduction of fracture energy, accompanied by a slight increase of elastic modulus, in agreement with earlier works (Currey, 1988a; Lucksanasombool et al., 2001; Nyman et al., 2006; Wang and Agrawal, 1996). The vascular porosity (Table 3) can be used to estimate the content of free water. According to early works (Evans, 1973), the total volume fraction of lacunae and canaliculi of cortical bone is less than the vascular porosity. Hence, assuming that the lacunae and canaliculi represented an average volume fraction equal to that of vascular pores (i.e., $3.75 \%$ in Table 3), then a majorant of average volume fraction of free water is $7.5 \%$ (i.e., $3.75 \%$ from vascular porosity plus the assumed $3.75 \%$ from lacunae and canaliculi). Thus, and taking into account the measured volume fraction of water (Table 2), the thermal dehydration removes a significant amount of bound water from the extracellular matrix, including the collagen. It is conceivable that removing water from the collagen underlies the observed changes on both fracture energy and elastic modulus (Nomura et al., 1977). The higher stiffness and lesser viscoelasticity of dehydrated collagen reduces the extension of the FPZ in the vicinity of crack tip and the energy consumption of related toughening events, causing a transition of fracture behaviour of cortical bone from ductile to brittle (Figs. 9 and 10), conjointly with a strong reduction of fracture energy (Tables 4 and 5). The minor influence of dehydration on the elastic modulus of cortical bone (Tables 4 and 5) can be explained by the different values of the elastic modulus of collagen and hydroxyapatite, which are in the order of $1.5 \mathrm{GPa}$ and $114 \mathrm{GPa}$, respectively (Zioupos et al., 1999).

\section{Conclusions}

In this work fracture of cortical bone tissue from young bovine femur under mode I loading was analysed. The double cantilever beam test was used with success. This constitutes an important result owing to significant advantages that this test presents relatively to other fracture tests for mode I fracture characterization. In addition, a new data reduction scheme based on the specimen compliance and crack equivalent concept was applied. The compliance based beam model does not depend on crack measurements during its growth and allows the attainment of the entire R-curve, thus leading to clear identification of fracture energy. The proposed methodology was validated numerically using a cohesive zone model 

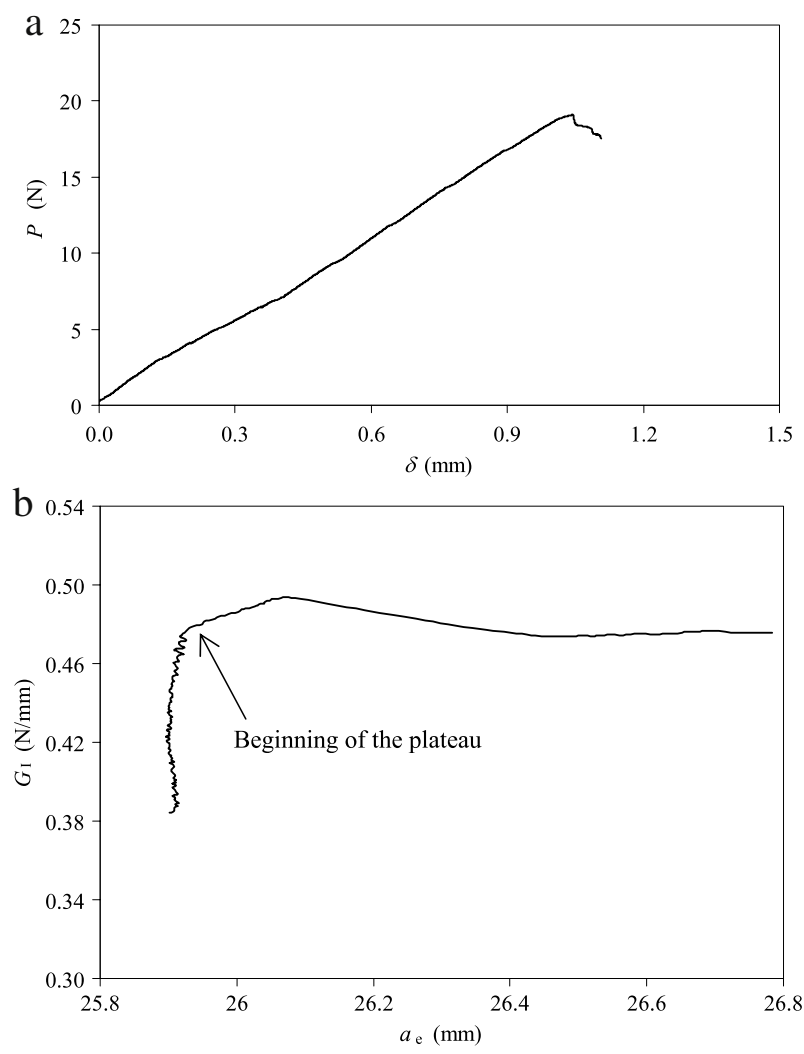

Fig. 10 - Typical P- $\delta$ (a) and the corresponding R-curve (b) for the dehydrated bone.

Table 4 - Fracture test results from hydrated cortical bone.

\begin{tabular}{|c|c|c|}
\hline \multirow[t]{2}{*}{ Specimen } & \multicolumn{2}{|c|}{ Experimental } \\
\hline & $E_{L}(\mathrm{GPa})$ & $\mathrm{G}_{\mathrm{Ic}}(\mathrm{N} / \mathrm{mm})$ \\
\hline 1 & 19.498 & 1.606 \\
\hline 2 & 16.659 & 1.690 \\
\hline 3 & 17.323 & 2.224 \\
\hline 4 & 17.984 & 2.133 \\
\hline 5 & 22.375 & 1.524 \\
\hline 6 & 19.857 & 2.050 \\
\hline 7 & 21.098 & 1.715 \\
\hline 8 & 21.364 & 2.367 \\
\hline 9 & 23.311 & 1.893 \\
\hline Average & 19.94 & 1.91 \\
\hline $\operatorname{Cov}(\%)$ & 11.53 & 15.53 \\
\hline
\end{tabular}

and demonstrated its accuracy to reproduce the inputted fracture energy. The experimental tests were performed on hydrated and dehydrated bone specimens, on the TL propagation system. The R-curves obtained using the proposed data reduction scheme clearly show that the dehydrated bone is much more brittle than the hydrated one. As a consequence, the fracture process zone of dehydrated bone is much less pronounced than in hydrated tissue, owing to the removal of water from the collagen matrix, which reduces the material ductility. The interaction between these two components (i.e., water and collagen matrix) contributes significantly to the fracture process and plays a fundamental role on the cortical bone fracture behaviour.
Table 5 - Fracture test results from dehydrated cortical

bone.

\begin{tabular}{|c|c|c|}
\hline \multirow[t]{2}{*}{ Specimen } & \multicolumn{2}{|c|}{ Experimental } \\
\hline & $E_{L}(\mathrm{GPa})$ & $\mathrm{G}_{\text {Ic }}(\mathrm{N} / \mathrm{mm})$ \\
\hline 1 & 23.511 & 0.592 \\
\hline 2 & 23.084 & 0.603 \\
\hline 3 & 23.726 & 0.510 \\
\hline 4 & 18.95 & 0.575 \\
\hline 5 & 25.681 & 0.486 \\
\hline 6 & 21.986 & 0.623 \\
\hline 7 & 23.035 & 0.477 \\
\hline 8 & 21.109 & 0.487 \\
\hline 9 & 22.52 & 0.518 \\
\hline 10 & 19.765 & 0.617 \\
\hline Average & 22.34 & 0.55 \\
\hline $\operatorname{Cov}(\%)$ & 8.86 & 10.70 \\
\hline
\end{tabular}

The principal achievements of this work confirm the applicability of the double cantilever beam test applying the data reduction method described to determine the fracture energy of bone under mode I loading.

\section{Acknowledgement}

The authors thank the Portuguese Foundation for Science and Technology for supporting the work here presented, through the research project PTDC/EME-PME/71273/2006.

\section{Appendix}

Eq. (3) can be expressed as,

$\alpha a_{e}^{3}+\beta a_{e}+\gamma=0$

where the coefficients $\alpha, \beta$ and $\gamma$ are, respectively

$\alpha=\frac{8}{B h^{3} E_{L}} ; \quad \beta=\frac{12}{5 B h G_{L R}} ; \quad \gamma=-C$.

Using the Matlab ${ }^{\circledR}$ software and only keeping the real solution it gives,

$a_{e}=\frac{1}{6 \alpha} \mathrm{A}-\frac{2 \beta}{\mathrm{A}}$

with

$A=\left(\left(-108 \gamma+12 \sqrt{3\left(\frac{4 \beta^{3}+27 \gamma^{2} \alpha}{\alpha}\right)}\right) \alpha^{2}\right)^{\frac{1}{3}}$

R E F E R E N C E S

Bowman, S.M., Gibson, L.J., Hayes, W.C., McMahon, T.A., 1999. Results from demineralized bone creep tests suggest that collagen is responsible for the creep behaviour of bone. Journal of Biomechanical Engineering 121, 253-258.

Brown, C.U., Yeni, Y.N., Norman, T.L., 2000. Fracture toughness is dependent on bone location - a study of the femoral neck, femoral shaft, and the tibial shaft. Journal of Biomedical Materials Research 49, 380-389.

Cox, B.N., Yang, Q., 2007. Cohesive zone models of localization and fracture in bone. Engineering Fracture Mechanics 74, 1079-1092. 
Currey, J.D., 1988a. The effects of drying and re-wetting on some mechanical properties of cortical bone. Journal of Biomechanics 5, 439-441.

Currey, J.D., 1988b. The effect of porosity and mineral content on the Young's modulus of elasticity of compact bone. Journal of Biomechanics 21, 131-139.

de Moura, M.F.S.F., Gonçalves, J.P.M., Marques, A.T., de Castro, P.M.S.T., 1997. Modelling compression failure after low velocity impact on laminated composites using interface elements. Journal of Composite Materials 31, 1462-1479.

de Moura, M.F.S.F., Morais, J., Dourado, N., 2008. A new data reduction scheme for mode I wood fracture characterization using the double cantilever beam test. Engineering Fracture Mechanics 75, 3852-3865.

Dempster, W.T., Coleman, R.F., 1961. Tensile strength of bone along and across the grain. Journal of Applied Physiology 16, 355-360.

Doblaré, M., García, J.M., Gómez, M.J., 2004. Modelling bone tissue fracture and healing: a review. Engineering Fracture Mechanics 71, 1809-1840.

Dong, X.N., Guo, X.E., 2004. The dependence of transversely isotropic elasticity of human femoral cortical bone on porosity. Journal of Biomechanics 37, 1281-1287.

Evans, F.G., 1973. Mechanical Properties of Bone. Charles C Thomas Publisher, Springfield, IL.

Hernandez, C.J., Beaupré, G.S., Keller, T.S., Carter, D.R., 2001. The influence of bone volume fraction and ash fraction on bone strength and modulus. Bone 29, 74-78.

ISO 15024:2001. Fibre-reinforced plastic composites - determination of mode I interlaminar fracture toughness, $G_{I c}$, for unidirectionally reinforced materials.

Lucksanasombool, P., Higgs, W.A.J., Higgs, R.J.E.D., Swain, M.V., 2001. Fracture toughness of bovine bone: influence of orientation and storage media. Biomaterials 22, 3127-3132.

Malik, C.L., Stover, S.M., Martin, R.B., Gibeling, J.C., 2003. Equine cortical bone exhibits rising $R$-curve fracture mechanics. Journal of Biomechanics 36, 191-198.

Morais, A.B., de Moura, M.F.S.F., Gonçalves, J.P.M., Camanho, P.P., 2003. Analysis of crack propagation in double cantilever beam tests of multidirectional laminates. Mechanics of Materials 35, 641-652.

Nalla, R.K., Kinney, J.H., Ritchie, R.O., 2003. Mechanistic fracture criteria for the failure of human cortical bone. Nature Materials 2, 164-168.

Nalla, R.K., Kruzic, J.J., Ritchie, R.O., 2004a. On the origin of the toughness of mineralized tissue: microcracking or crack bridging? Bone 34, 790-798.

Nalla, R.K., Kruzic, J.J., Kinney, J.H., Ritchie, R.O., 2004b. Effect of aging on the toughness of human cortical bone: evaluation by R-curves. Bone 35, 1240-1246.

Nomura, S., Hiltner, A., Lando, J.B., Baer, E., 1977. Interaction of water with native collagen. Biopolymers 2, 231-246.

Norman, T.L., Nivargikar, S.V., Burr, D.B., 1996. Resistance to crack growth in human cortical bone is greater in shear than in tension. Journal of Biomechanics 29, 1023-1031.

Norman, T.L., Vashishth, D., Burr, D.B., 1995. Fracture toughness of human bone under tension. Journal of Biomechanics 28, 309-320.
Nyman, J.S., Roy, A., Shen, X., Acuna, R.L., Tyler, J.H., Wang, X., 2006. The influence of water removal on the strength and toughness of cortical bone. Journal of Biomechanics 39, 931-938.

Phelps, J.B., Hubbard, G.B., Wang, X., Agrawal, C.M., 2000. Microstructural heterogeneity and the fracture toughness of bone. Journal of Biomedical Materials Research Part A 51, 735-741.

Rho, J.-Y., Kuhn-Spearing, L., Zioupos, P., 1998. Mechanical properties and the hierarchical structure of bone. Medical Engineering \& Physics 20, 92-102.

Sasaki, N., Enyo, A., 1995. Viscoelastic properties of bone as a function of water content. Journal of Biomechanics 28, 809-815.

Ural, A., Vashishth, D., 2006. Cohesive finite element modelling of age-related toughness loss in human cortical bone. Journal of Biomechanics 39, 2974-2982.

Vashishth, D., 2004. Rising crack-growth-resistance behaviour in cortical bone: implications for toughness measurements. Journal of Biomechanics 37, 943-946.

Vashishth, D., Tanner, K.E., Bonfield, W., 2000. Contribution, development and morphology of microcracking in cortical bone during crack propagation. Journal of Biomechanics 33, 1169-1174.

Wang, X., Agrawal, C.M., 1996. Fracture toughness of bone using a compact sandwich specimen: effects of sampling sites and crack orientation. Journal of Biomedical Materials Research Part B: Applied Biomaterials 33, 13-21.

Wang, X., Bank, R.A., TeKoppele, J.M., Hubbard, G.B., Athanasiou, K.A., Agrawal, C.M., 2000. Effect of collagen denaturation on the toughness of bone. Clinical Orthopaedics and Related Research 371, 228-239.

Wang, X., Puram, S., 2004. The toughness of cortical bone and its relationship with age. Annals of Biomedical Engineering 32, 123-135.

Wang, X., Shen, X., Li, X., Agrawal, M., 2002. Age-related changes in the collagen network and toughness of bone. Bone 31, 1-7.

Yamashita, J., Li, X., Furman, B.R., Rawls, H.R., Wang, X., Agrawal, C.M., 2002. Collagen and bone viscoelasticity: a dynamic mechanical analysis. Journal of Biomedical Materials Research Part B: Applied Biomaterials 63, 31-36.

Yan, J., Clifton, K.B., Mecholsky, J.J., Reep, R.L., 2006. Fracture toughness of manatee rib and bovine femur using a chevronnotched beam test. Journal of Biomechanics 39, 1066-1074.

Yang, Q.D., Cox, B.N., Nalla, R.K., Ritchie, R.O., 2006. Re-evaluating the toughness of human cortical bone. Bone 38, 878-887.

Yeni, Y.N., Norman, T.L., 2000. Calculation of porosity and osteonal cement line effects on the effective fracture toughness of cortical bone in longitudinal crack growth. Journal of Biomedical Materials Research Part B: Applied Biomaterials 51, 504-509.

Zioupos, P., 1998. Recent developments in the study of failure of solid biomaterials and bone: fracture and pre-fracture toughness. Materials Science \& Engineering C 6, 33-40.

Zioupos, P., Currey, J.C., Hamer, A.J., 1999. The role of collagen in the declining mechanical properties of ageing human cortical bone. Journal of Biomedical Materials Research Part A 45, 108-116. 